Check for updates

Cite this: RSC Adv., 2018, 8, 6306

Received 6th January 2018

Accepted 22nd January 2018

DOI: $10.1039 / \mathrm{c} 8 \mathrm{ra} 00161 \mathrm{~h}$

rsc.li/rsc-advances

\section{Design, synthesis, insecticidal activity and 3D-QSR study for novel trifluoromethyl pyridine derivatives containing an 1,3,4-oxadiazole moiety $\dagger$}

\begin{abstract}
F. Z. Xu, ${ }^{a}$ Y. Y. Wang,,$^{a}$ D. X. Luo, ${ }^{a}$ G. Yu, ${ }^{a}$ S. X. Guo, ${ }^{a} H . F u,{ }^{a}$ Y. H. Zhao ${ }^{b}$ and J. Wu (D) *a
A series of trifluoromethyl pyridine derivatives containing 1,3,4-oxadiazole moiety was designed, synthesized and bio-assayed for their insecticidal activity. The result of bio-assays indicated the synthesized compounds exhibited good insecticidal activity against Mythimna separata and Plutella xylostella, most of the title compounds show $100 \%$ insecticidal activity at $500 \mathrm{mg} \mathrm{L}^{-1}$ and $>80 \%$ activity at $250 \mathrm{mg} \mathrm{L}^{-1}$ against the two pests. Compounds E18 and E27 showed LC 50 values of 38.5 and $30.8 \mathrm{mg} \mathrm{L}^{-1}$ against Mythimna separata, respectively, which were close to that of avermectin (29.6 $\mathrm{mg} \mathrm{L}^{-1}$ ); compounds E5, E6, E9, E10, E15, E25, E26, and E27 showed 100\% activity at $250 \mathrm{mg} \mathrm{L}^{-1}$, which were better than chlorpyrifos (87\%). COMFA and COMSIA models with good predictability were proposed, which revealed the electron-withdrawing groups with an appropriate bulk at 2- and 4positions of benzene ring could enhance insecticidal activity.
\end{abstract}

\section{Introduction}

Harmful insect pest in agriculture has brought about actual losses in productivity of crops all over the world annually. ${ }^{1}$ Take Mythimna separata Walker and Plutella xylostella as examples, they cause enormous economic losses in agricultural production worldwide. ${ }^{2,3}$ Currently, because of the generation of resistance and cross resistance, the insecticidal effect of phthaldiamides (such as fluobendiamide) and traditional organophosphorus pesticides was tapered. ${ }^{4-8}$ Thus, the development of insecticidal agent with novel structure is increasingly required.

Pesticide containing the fluorine is the hot topics in creating novel pesticides. ${ }^{9}$ As an important class of fluorinated heterocycle, the skeleton of trifluoromethyl pyridine showed a vital role in the discovery of pesticide molecules; so far, there are about 27 commercial pesticides with trifluoromethyl pyridine were commercialized, and five of them were used as insecticidal agent (Fig. 1). ${ }^{9}$ In recent years, studies for development of novel potential insecticidal molecules with the trifluoromethyl

${ }^{a}$ State Key Laboratory Breeding Base of Green Pesticide and Agricultural Bioengineering, Key Laboratory of Green Pesticide and Agricultural Bioengineering, Ministry of Education, Research and Development Center for Fine Chemicals, Guizhou University, Huaxi District, Guiyang 550025, P. R. China. E-mail: wujian2691@126.com; jwu6@gzu.edu.cn

${ }^{b}$ Institute for the Control of Agrochemicals, Ministry of Agriculture, Beijing 100125, China

$\dagger$ Electronic supplementary information (ESI) available: The copies of ${ }^{1} \mathrm{H}$ NMR, ${ }^{19} \mathrm{~F}$ NMR ${ }^{13} \mathrm{C}$ NMR and HR-MS spectrograms for all the synthesized compounds can be found in the ESI. See DOI: $10.1039 / \mathrm{c} 8 \mathrm{ra00161h}$

\$ Co-first author for the manuscript. pyridine are very popular, and large number of potential compounds with this substructure were reported. ${ }^{10-16}$ Such as sulfoxaflor, a representative commercial insecticide, and was industrialized by Dow Agroscience. ${ }^{16}$

1,3,4-Oxadiazole derivative is a highly active pharmacophore and widely was used in pesticide molecules, ${ }^{17}$ many active substance containing such a scaffold have been reported as insecticides, ${ }^{18-24}$ fungicides, ${ }^{25-31}$ and herbicides, ${ }^{32-34}$ some of them were treated as a pesticide candidate for further industrialization. ${ }^{25}$ Recently, Liu and co-workers reported the 1,3,4oxadiazole derivative with an anthranilic diamide moiety possessed excellent insecticidal activity against $P$. xylostella ${ }^{20}$ as well as some insecticidal sarisan analogues with an 1,3,4-oxadiazole scaffold were developed by Guo et al. ${ }^{23,35}$ And in our recent work, a series of insecticidal 1,3,4-oxadiazole molecules bearing a 3-chloropyridin-2-yl-1 $H$-pyrazole was proposed. ${ }^{36}$

Inspired by the description above, herein we attempted to replace 3-chloropyridin-2-yl- $1 H$-pyrazole using a trifluorom-

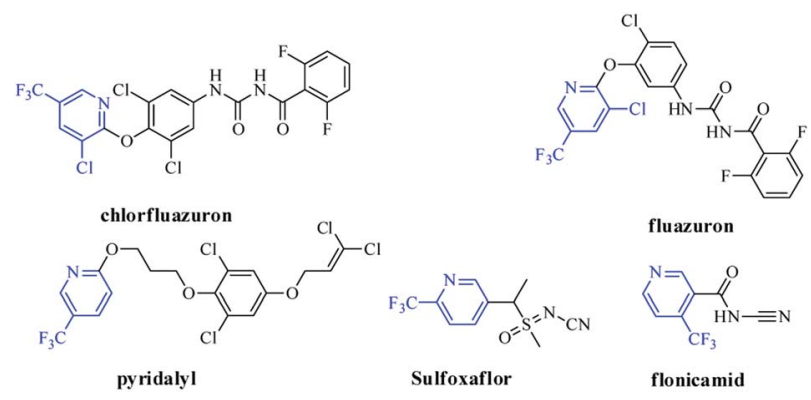

Fig. 1 The commercial insecticide containing trifluoromethyl pyridine. 


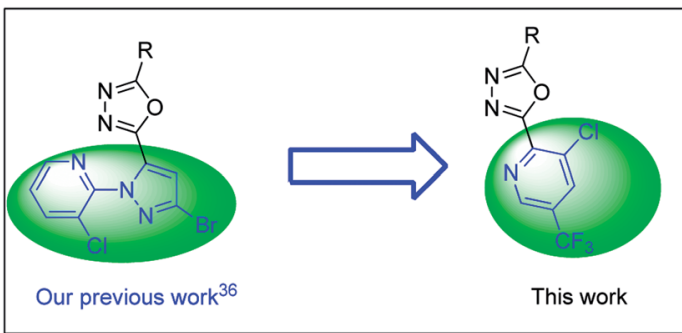

Fig. 2 The design of title compound.

ethyl pyridine based on our previous work (Fig. 2), ${ }^{36}$ then structural variation at the other side of the 1,3,4-oxadiazole ring by the introduction of different substituted phenyl, resulting in series of trifluoromethyl pyridine derivatives containing an 1,3,4-oxadiazole moiety. Their insecticidal activity was evaluated, as well as the quantitative structure-activity relationship was analyzed based on CoMFA and CoMSIA. To the best of our knowledge, this is the first report on the insecticidal activity of 2-(trifluoromethyl pyridin-2-yl)-1,3,4-oxadiazole derivatives.

\section{Results and discussion}

\section{Chemistry}

The synthetic route for the title compounds is depicted in Scheme 1. The key intermediate ethyl substituted benzoate (B) was easily obtained in good yield via reactions of substituted benzoic acid with ethanol in the present of concentrated sulfuric acid, which further reacted with hydrazine hydrate $(80 \%)$ to yield substituted benzohydrazide $\mathrm{C}$ in $>90 \%$ yields. ${ }^{25,37}$ Subsequently, treatment of $\mathbf{C}$ with 3-chloro-5-(trifluoromethyl) picolinic acid (D) in the presence of phosphorus oxychloride at refluxing temperature afforded the title compounda (E) with excellent yield by employed known protocol. ${ }^{36}$

Structures of the compounds E1 to E28 were established on basis of ${ }^{1} \mathrm{H}$ NMR, ${ }^{19} \mathrm{~F}$ NMR, ${ }^{13} \mathrm{C}$ NMR and HR-MS data. In the ${ }^{1} \mathrm{H}$ NMR spectra, the $\mathrm{H}$ proton near the " $N$ " of pyridine appeared as a singlet near $\delta 8.95 \mathrm{ppm}$. As well as the proton at ortho-position of chlorine atom at pyridine appeared as a singlet near $\delta$ 8.20. In

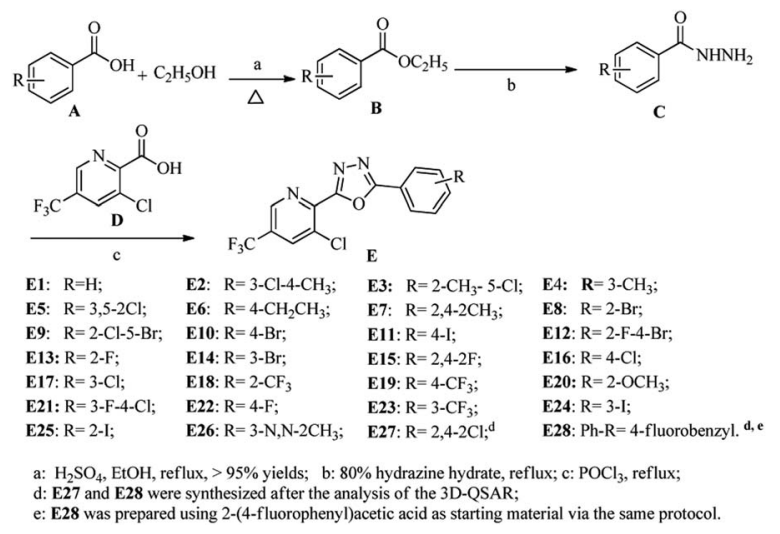

Scheme 1 The synthetic routine of the title compounds E1-E28. its ${ }^{13} \mathrm{C}$ NMR spectra, the carbons near the group of " $-\mathrm{CF}_{3}$ " were split into a quartet due to the coupling coefficients from " $F$ ", take compound $\mathbf{E 1}$ as example, the carbon in " $-\mathrm{CF}_{3}$ " group is near $\delta_{\mathrm{C}} 122.21 \mathrm{ppm}$ and the coupling constant $\left({ }^{1} J_{\mathrm{F}-\mathrm{C}}\right)$ was $273.5 \mathrm{~Hz}$, the carbon that is linked to group of " $-\mathrm{CF}_{3}$ " resonance frequency is near $\delta_{\mathrm{C}} 128.75 \mathrm{ppm}$ as a quartet and with the coupling constant $\left({ }^{2} J_{\mathrm{C}-\mathrm{F}}\right)$ is $34.1 \mathrm{~Hz}$; and the carbons at orthoposition of " $-\mathrm{CF}_{3}$ " were also split into very weak quartets with coupling constant $\left({ }^{3} J_{\mathrm{C}-\mathrm{F}}\right)$ ranged from 3.6 to $3.8 \mathrm{~Hz}$.

\section{Insecticidal activity and 3D-QSAR study}

The insecticidal activity of the synthesized compound against Mythimna separata (Walker) was carried out using reference method..$^{38}$ The result listed in Table 1 revealed most of the synthesized compounds showed $100 \%$ activity against $M$. separata (Walker) at the $500 \mathrm{mg} \mathrm{L}^{-1}$. And the most of them displayed $>80 \%$ activity at $250 \mathrm{mg} \mathrm{L}{ }^{-1}$. The median lethal concentrations $\left(\mathrm{LC}_{50}\right)$ values for all of the synthesized compounds were further investigated and also shown in Table 1. For demonstrating the effectiveness of evaluation, the $\mathrm{LC}_{50}$ value of commercial avermectin (a commonly used insecticide) was also evaluated as positive control. The results indicated the $\mathrm{LC}_{50}$ of synthesized compound was ranged from $38.0 \mathrm{mg} \mathrm{L}^{-1}$ to $284.6 \mathrm{mg} \mathrm{L}^{-1}$, in particularly, the $\mathrm{LC}_{50}$ values of compounds $\mathbf{E 1 8}$ and $\mathbf{E} 27$ were 38.5 and $30.8 \mathrm{mg} \mathrm{L}^{-1}$, respectively. And the $\mathrm{LC}_{50}$ value of E27 was the similar as that of avermectin $\left(29.6 \mathrm{mg} \mathrm{L}^{-1}\right)$. A preliminary SAR study indicated that the insecticidal activity can be impacted by the different type of groups on benzene ring, it could be concluded that the activity could be enhanced by introduction of the electron-withdrawing groups, and decreased by electron-donating group. For example, the activity of compounds with a methyl (E2, E3, E4, E7) and ethyl (E6) showed much lower activity than and the compounds with an electronwithdrawing group (such as E8, E18, E27), and the introduction of electronegative group could increase the insecticidal activity.

In order to give more information of structure-activity relationship, 3D-QSAR models (CoMFA and CoMSIA) were further built based on the active data of M. separata (Walker). Six compounds (E7, E8, E9, E16, E22 and E26) were randomly chosen as a test set. The training set consisted of 20 compounds. The superimposition of training set compounds was done by using compounds E20 as a template and had shown in ESI (Fig. 1S†).

The results of the PLS statistics tested on bioactivity against M. separata are presented in Table S1 (see ESI $\dagger$ ). In result of CoMFA, the cross-validated $q^{2}$ value was 0.742 with five components, and the non-crossvalidated conventional $r^{2}$ value was 0.975 with a standard error of estimation (SEE) of 0.044 and $F=92.362$. The relative contributions between steric and electrostatic fields for the CoMFA model were 0.586 and 0.414, respectively. As for CoMSIA, as well as the $q^{2}$ value and optimum number of components were 0.614 and 6 , respectively. And the $r^{2}$ value, SEE, and $F$ value were $0.92,0.084$, and 32.793 , respectively. And the CoMSIA result revealed the contributions of steric, electrostatic hydrophobic, hydrogen-bond donor and acceptor fields were $0.511,0.489,0,0$, and 0 , respectively. These 
Table 1 Insecticidal activity of title compounds against Mythimna separata (Walker)

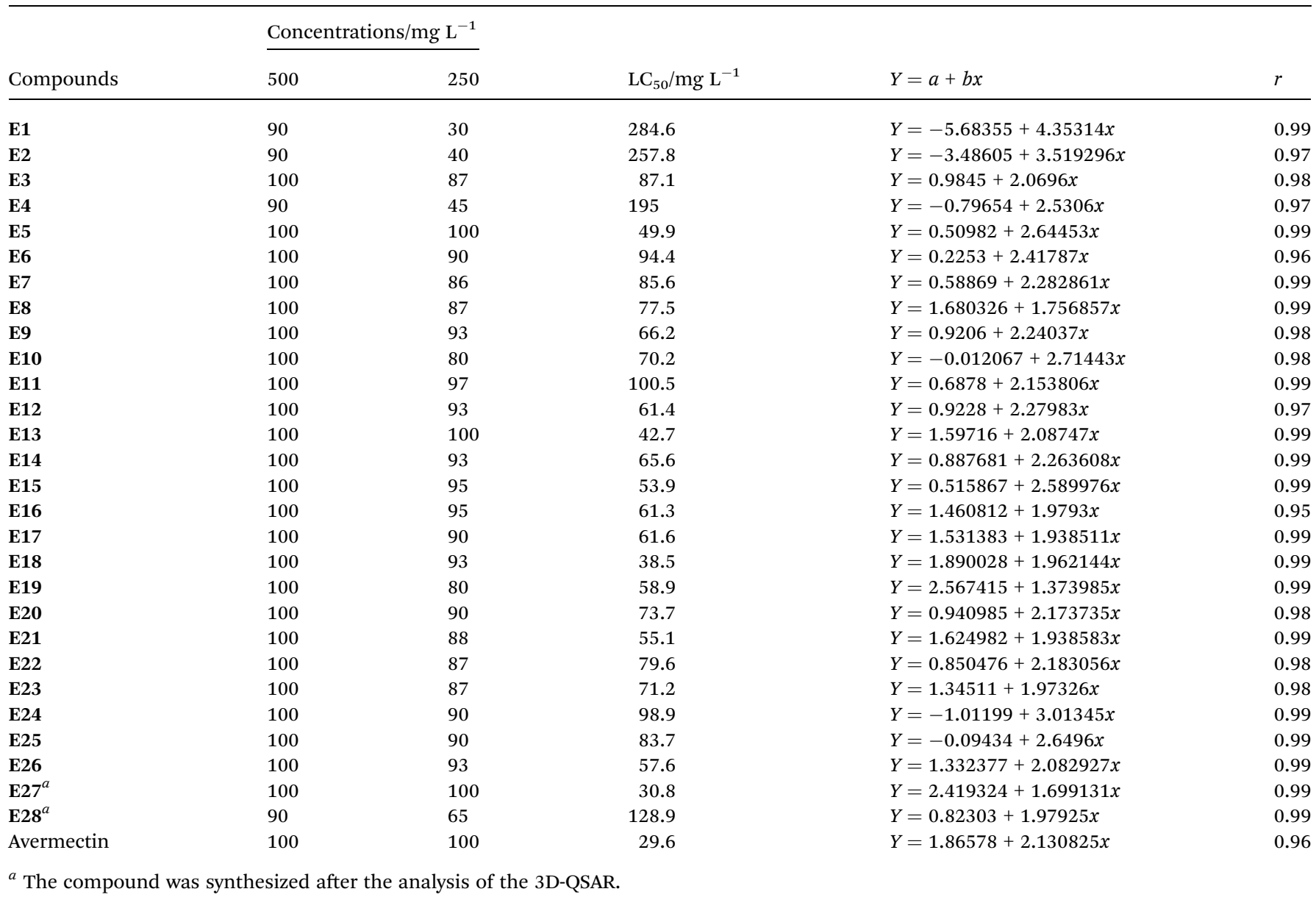

results indicated that both CoMFA and CoMSIA models showed good predictability. As well as the two models revealed that both the steric and electrostatic field made an important contribution to bioactivity, and steric field show a little priority than electrostatic field.

The PLC $_{50}$ values of the training set compounds and test set compounds were predicted by the two models (more details can be found in ESI, Table S2 $\dagger$ ). And the correlations between the predicted $\mathrm{PLC}_{50}$ and experimental $\mathrm{PLC}_{50}$ are represented in Fig. 3 . The result indicated the proposed model predicted the activity successfully.

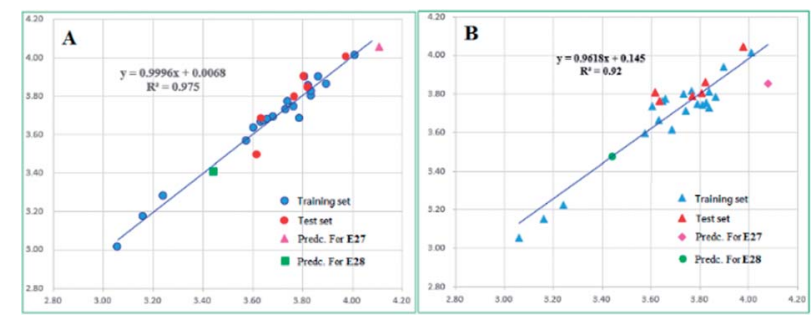

Fig. 3 Scatter plots between experimental activities and predicted activities for COMFA (A) and CoMSIA (B).
The steric contour map of CoMFA is shown in Fig. 4(A and B). The green contours near the 2- and 4-positions of benzene ring revealed a large substituent is necessary for contributing to the anti-insecticidal activity, however, the yellow contours near the two positions indicated a bulky substituent could be decreased the activity, for instance, the activity of compound $\mathbf{E 6}$ (with ethyl at 4-position of benzene and $\mathrm{LC}_{50}=94.4 \mathrm{mg} \mathrm{L}^{-1}$ ), and $\mathbf{E 2 0}$ (with ethyl at 2-position of benzene and $\mathrm{LC}_{50}=73.7 \mathrm{mg} \mathrm{\textrm {L } ^ { - 1 }}$ ) was much low than $\mathbf{E 2 2}\left(\mathrm{LC}_{50}=79.6 \mathrm{mg} \mathrm{L}^{-1}\right)$ and $\mathbf{E 8}\left(\mathrm{LC}_{50}=\right.$

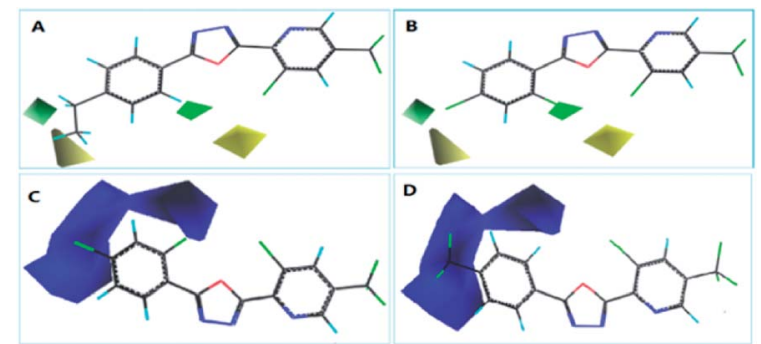

Fig. 4 The contour map of CoMFA. (A) Steric contour map with compound E6, (B) steric contour map with compound E12; (C) electrostatic contour map with compound E12, (D) electrostatic contour map with compound E19. 


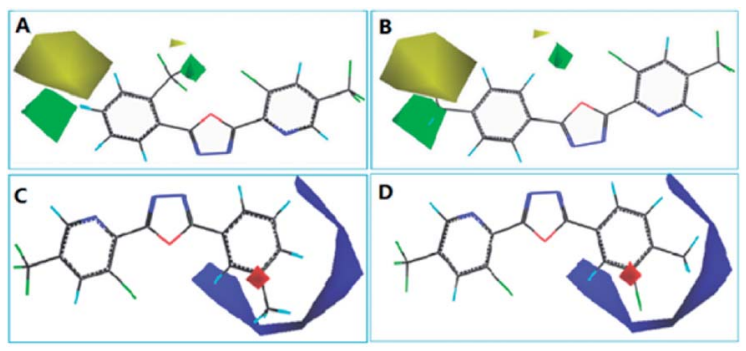

Fig. 5 The contour map of COMSIA. (A) Steric contour map with compound E18, (B) steric contour map with compound E6; (C) electrostatic contour map with compound E4, (D) electrostatic contour map with compound E2.

$42.7 \mathrm{mg} \mathrm{L}^{-1}$ ), thus an appropriate size of substituent should be considered near the two positions, from the $\mathrm{LC}_{50}$ values of the compounds, we speculated a chlorine at 4-position of benzene (E16, $\mathrm{LC}_{50}=61.3 \mathrm{mg} \mathrm{L}^{-1}$ ) is much more suitable than other halogen atoms. Moreover, in electrostatic contour map (Fig. 4C and $\mathrm{D}$ ), the blue contour indicated that negative charge near the benzene ring (2-5 positions) is favored by the activity. Hence, the electron withdrawing group is in favor of improving the insecticidal activity, the activity for the compounds with an electron-donating group is much lower than these compounds containing an electron withdrawing group (e.g.: E4 $<$ E23, E24 et al.; E6 < E22, E7 < E12).

The CoMSIA contour map was shown in Fig. 5, the steric and electrostatic contour map (Fig. 5A and B) revealed the similar regularity as CoMFA shown. And furthermore, the electrostatic contour map (Fig. 5C and D) give a necessary supplement for the electrostatic contour map of CoMFA, from the result of CoMSIA, we can see that the electronegative groups around the benzene is necessary, but an appropriate group at 3-position of benzene should be taken into account, or else the activity was be decreased (e.g.: the $\mathrm{LC}_{50}$ value of compound $\mathbf{E} 2$ is $\left.257.8 \mathrm{mg} \mathrm{L}^{-1}\right)$. Moreover, the predictability of the 3D-QSAR models were investigated by the new structures with two chlorine atoms (E27) and a 4-fluorobenzyl (E28), which were synthesized and tested for their insecticidal activities, the results showed in Table $\mathrm{S} 2 \uparrow$ indicated the models showed good predictability.

The insecticidal activity of the synthesized compounds against Plutella xylostella, Nilaparvata lugens and Aphis craccivora Koch were carried out using reference method. ${ }^{39,40}$ The results listed in Table 2 indicated that some of the synthesized compounds showed certain insecticidal activity against Nilaparvata lugens and Aphis craccivora Koch at $500 \mathrm{mg} \mathrm{L}^{-1}$, the activity of compounds E14, E15 and E18 was medium. However, the synthesized compounds showed good insecticidal against Plutella xylostella. Most of them showed $100 \%$ activity at $500 \mathrm{mg} \mathrm{L}^{-1}$ and $>80 \%$ at $250 \mathrm{mg} \mathrm{L}^{-1}$, and in particularly, compounds E5, E6, E9, E10, E15, E25, E26, and E27 showed $100 \%$ activity, which were better than chlorpyrifos $(87 \%)$. The preliminary SAR analysis show similar trends as that of $M$. separata.
Table 2 Insecticidal activity of title compounds against $P$. xylostella, $N$. lugens, and A. craccivor

\begin{tabular}{|c|c|c|c|c|}
\hline \multirow[b]{2}{*}{ Compounds } & \multicolumn{2}{|l|}{ P. xylostella } & \multirow{2}{*}{$\frac{N . \text { lugens }}{500 \mathrm{mg} \mathrm{L}^{-1}}$} & \multirow{2}{*}{$\frac{\text { A. craccivora }}{500 \mathrm{mg} \mathrm{L}^{-1}}$} \\
\hline & $500 \mathrm{mg} \mathrm{L}^{-1}$ & $250 \mathrm{mg} \mathrm{L}^{-1}$ & & \\
\hline E1 & 100 & 63 & 20 & 20 \\
\hline E2 & 100 & 60 & 50 & 10 \\
\hline E3 & 100 & 60 & 30 & 50 \\
\hline E4 & 100 & 87 & 40 & 10 \\
\hline E5 & 100 & 100 & 20 & 40 \\
\hline E6 & 100 & 100 & 20 & 10 \\
\hline E7 & 100 & 80 & 50 & 40 \\
\hline E8 & 100 & 90 & 45 & 44 \\
\hline E9 & 100 & 100 & 63 & 55 \\
\hline E10 & 100 & 100 & 20 & 20 \\
\hline E11 & 100 & 93 & 30 & 30 \\
\hline E12 & 100 & 97 & 32 & 42 \\
\hline E13 & 100 & 90 & 10 & 60 \\
\hline E14 & 100 & 93 & 50 & 50 \\
\hline E15 & 100 & 100 & 60 & 65 \\
\hline E16 & 100 & 67 & 10 & 40 \\
\hline E17 & 100 & 87 & 14 & 44 \\
\hline E18 & 100 & 93 & 54 & 54 \\
\hline E19 & 100 & 90 & 23 & 0 \\
\hline E20 & 100 & 85 & 15 & 0 \\
\hline E21 & 96 & 70 & 13 & 15 \\
\hline E22 & 87 & 90 & 29 & 35 \\
\hline E23 & 100 & 97 & 24 & 40 \\
\hline E24 & 100 & 93 & 30 & 40 \\
\hline E25 & 100 & 100 & 30 & 23 \\
\hline E26 & 100 & 100 & 15 & 41 \\
\hline E27 & 100 & 100 & 23 & 43 \\
\hline E28 & 100 & 93 & 23 & 34 \\
\hline Chlorpyrifos & 100 & 87 & - & - \\
\hline Imidacloprid & - & - & 100 & 100 \\
\hline
\end{tabular}

\section{Conclusions}

In current work, a new series of 1,3,4-oxadiazole derivatives containing trifluoromethyl pyridine moiety was designed and synthesized. The structures of these compounds were characterized and confirmed by ${ }^{1} \mathrm{H}$ NMR, ${ }^{19} \mathrm{~F}$ NMR, ${ }^{13} \mathrm{C}$ NMR, HR-MS. Bioassays for insecticidal activity of the synthesized compounds against $M$. separata, $P$. xylostella, $N$. lugens, and A. craccivora were conducted. The results indicated some of the synthesized compounds exhibited good insecticidal activity against $M$. separata (with $\mathrm{LC}_{50}$ values ranged from $30.8 \mathrm{mg} \mathrm{\textrm {L } ^ { - 1 }}$ to $284.6 \mathrm{mg} \mathrm{L}^{-1}$ ) and P. xylostella (most of them showed $100 \%$ activity at $500 \mathrm{mg} \mathrm{L}^{-1}$ and $>80 \%$ at $250 \mathrm{mg} \mathrm{L}^{-1}$ ). In particularly, the $\mathrm{LC}_{50}$ value of compounds E18 and E27 was 38.5 and $30.8 \mathrm{mg} \mathrm{L}{ }^{-1}$, respectively. CoMFA and CoMSIA models were built, the models exhibited good correlation, predictability, and revealed the activity could be enhanced by introduction of the electron-withdrawing groups with proper bulk at 2- and 4positions of benzene ring. The proposed models could predict the activity with reasonable accuracy and will be useful in the near future for finding potent insecticidal molecules in our group. 


\section{Experimental section}

\section{Materials and methods}

All aromatic acids were purchased from Accela Chem-Bio Co., Ltd (Shanghai, China) and Inno-chem Co., Ltd (Beijing, China). Melting points of the synthesized compounds were measured using a XT-4 binocular microscope (Beijing Tech Instrument Co., China). ${ }^{1} \mathrm{H},{ }^{19} \mathrm{~F}$ and ${ }^{13} \mathrm{C}$ NMR spectra were recorded on a JEOL ECX 500 NMR (JEOL Ltd., Japan) or AVANCE III HD 400M NMR (Bruker Corporation, Switzerland) spectrometer operating at room temperature using $\mathrm{CDCl}^{3}$ or DMSO as solvents. HR-MS was recorded on an Orbitrap LC-MS instrument (Q-Exative, Thermo Scientific ${ }^{\mathrm{TM}}$, and American). The course of the reactions was monitored by TLC.

\section{Synthetic procedures}

Synthesis of substituted benzoyl hydrazine (C). Substituted benzoyl hydrazine (C) was prepared by following the known procedure. ${ }^{25}$ Substituted benzoic acid or para-fluorophenylacetic acid (for preparation of compound E28) as starting materials, followed by esterification and hydrazinolysis to yield substituted 2-phenoxyacetohydrazide in excellent yields. ${ }^{37}$

Synthesis of title compounds (E1 to E28). ${ }^{36}$ The mixture of substituted 2-phenoxyacetohydrazide C (0.5 mmol), 3-chloro-5(trifluoromethyl)picolinic acid $(0.5 \mathrm{mmol})$ and phosphorus oxychloride $(3 \mathrm{~mL})$ was stirred for $8 \mathrm{~h}$ under refluxing temperature, the resulted mixture was the poured to crushed ice and neutralized with $5 \% \mathrm{NaOH}$, the precipitate was then filtered and purification using silica gel (200-300 mesh) column chromatography with using ethyl acetate/petroleum ether $(1: 5)$. The melting point, yield, IR, ${ }^{1} \mathrm{H}$ NMR, ${ }^{13} \mathrm{C}$ NMR and HR-MS data for compound E1 are listed below, and those for compounds E2E28 can be found in the ESI. $\dagger$

2-(3-Chloro-5-(trifluoromethyl)pyridin-2-yl)-5-phenyl-1,3,4oxadiazole (E1). Yield 58.2\%; light yellow solid; mp 104.2$105.4{ }^{\circ} \mathrm{C} ;{ }^{1} \mathrm{H}$ NMR $(500 \mathrm{MHz}$, chloroform-D) $\delta 8.96(\mathrm{~d}, J=1.0 \mathrm{~Hz}$, $1 \mathrm{H}$, pyridine- $\mathrm{H}), 8.23-8.21(\mathrm{~m}, 1 \mathrm{H}$, pyridine $-\mathrm{H}), 8.20(\mathrm{dt}, J=3.6$, $1.9 \mathrm{~Hz}, 2 \mathrm{H}, \mathrm{Ph}-\mathrm{H}), 7.61-7.53$ (m, 3H, Ph-H); ${ }^{19} \mathrm{~F} \mathrm{NMR} \mathrm{(471} \mathrm{MHz,}$ chloroform-D) $\delta-62.41 ;{ }^{13} \mathrm{C}$ NMR (125 MHz, chloroform-D) $\delta 165.84,161.23,144.66$ (q, $J=3.8 \mathrm{~Hz}), 144.09,136.76$ (q, $J=$ $3.6 \mathrm{~Hz}), 132.57,132.18,129.30,128.75$ (q, $J=34.1 \mathrm{~Hz}), 127.60$, 123.25, 122.21 (q, $J=273.5 \mathrm{~Hz}$ ); HR-MS (ESI): calculated for $\mathrm{C}_{14} \mathrm{H}_{7} \mathrm{ClF}_{3} \mathrm{~N}_{3} \mathrm{O}[\mathrm{M}+\mathrm{H}]^{+}$: 326.03025, found: 326.02969 .

2-(3-Chloro-4-methylphenyl)-5-(3-chloro-5-(trifluoromethyl)pyridin-2-yl)-1,3,4-oxadiazole (E2). Yield 48.3\%; light yellow solid; mp 94.0-96.2 ${ }^{\circ} \mathrm{C} ;{ }^{1} \mathrm{H}$ NMR (500 MHz, chloroform-D) $\delta 8.96$ (s, $1 \mathrm{H}$, pyridine- $\mathrm{H}), 8.19(\mathrm{~d}, J=9.1 \mathrm{~Hz}, 2 \mathrm{H}$, pyridine- $\mathrm{H}), 8.00(\mathrm{~d}, J$ $=7.9 \mathrm{~Hz}, 1 \mathrm{H}, \mathrm{Ph}-\mathrm{H}), 7.41$ (d, $J=7.9 \mathrm{~Hz}, 1 \mathrm{H}, \mathrm{Ph}-\mathrm{H}), 2.47(\mathrm{~s}, 3 \mathrm{H}$, $\left.-\mathrm{CH}_{3}\right) ;{ }^{19} \mathrm{~F}$ NMR (471 MHz, chloroform-D) $\delta-62.43 ;{ }^{13} \mathrm{C}$ NMR (125 MHz, chloroform-D) $\delta 164.80,161.22,144.63$ (d, $J=3.5 \mathrm{~Hz}$ ), 143.93, 141.21, 136.76 (d, $J=3.3 \mathrm{~Hz}), 135.49,132.23,131.81$, $128.80(\mathrm{q}, J=34.2 \mathrm{~Hz}), 127.88,125.65,122.18(\mathrm{q}, J=273.5 \mathrm{~Hz})$, 122.31, 20.49; HR-MS (ESI): calculated for $\mathrm{C}_{15} \mathrm{H}_{8} \mathrm{Cl}_{2} \mathrm{~F}_{3} \mathrm{~N}_{3} \mathrm{O}[\mathrm{M}+$ $\mathrm{H}]^{+}$: 374.00693, found: 374.00598 .

2-(5-Chloro-2-methylphenyl)-5-(3-chloro-5-(trifluoromethyl)pyridin-2-yl)-1,3,4-oxadiazole (E3). Yield 29.0\%; light yellow solid; mp 128.4-131.5 ${ }^{\circ} \mathrm{C} ;{ }^{1} \mathrm{H}$ NMR (500 MHz, chloroform-D) $\delta 8.97(\mathrm{~s}, 1 \mathrm{H}$, pyridine-H), $8.20(\mathrm{~s}, 1 \mathrm{H}$, pyridine- $\mathrm{H}), 8.09(\mathrm{~s}, 1 \mathrm{H}$, $\mathrm{Ph}-\mathrm{H}$ ), 7.43 (d, $J=8.3 \mathrm{~Hz}, 1 \mathrm{H}, \mathrm{Ph}-\mathrm{H}), 7.33$ (d, $J=8.1 \mathrm{~Hz}, 1 \mathrm{H}, \mathrm{Ph}-$ $\mathrm{H}), 2.77\left(\mathrm{~s}, 3 \mathrm{H},-\mathrm{CH}_{3}\right) ;{ }^{19} \mathrm{~F}$ NMR (471 MHz, chloroform-D) $\delta-62.43 ;{ }^{13} \mathrm{C}$ NMR (125 MHz, chloroform-D) $\delta$ 164.94, 160.99, $144.72(\mathrm{~d}, J=3.5 \mathrm{~Hz}), 143.98,137.62,136.75(\mathrm{~d}, J=3.3 \mathrm{~Hz})$, 133.40, 132.31, 132.23, 131.88, 129.11, 128.90 (q, $J=34.2 \mathrm{~Hz}$ ), 123.74, 122.19 (q, $J=273.8 \mathrm{~Hz}$ ), 21.84; HR-MS (ESI): calculated for $\mathrm{C}_{15} \mathrm{H}_{8} \mathrm{Cl}_{2} \mathrm{~F}_{3} \mathrm{~N}_{3} \mathrm{O}[\mathrm{M}+\mathrm{H}]^{+}: 374.00693$, found: 374.00607 .

2-(3-Chloro-5-(trifluoromethyl)pyridin-2-yl)-5-(m-tolyl)-1,3,4oxadiazole (E4). Yield 61.1\%; light red solid; mp $75.4-76.6{ }^{\circ} \mathrm{C}$; ${ }^{1} \mathrm{H}$ NMR (500 MHz, chloroform-D) $\delta 8.96(\mathrm{dd}, J=1.7,0.7 \mathrm{~Hz}, 1 \mathrm{H}$, pyridine-H), 8.19 (dd, $J=1.8,0.6 \mathrm{~Hz}, 1 \mathrm{H}$, pyridine- $\mathrm{H}$ ), 8.01 (ddd, $J=8.7,4.4,0.9 \mathrm{~Hz}, 2 \mathrm{H}, \mathrm{Ph}-\mathrm{H}), 7.41$ (dt, $J=15.2,4.0 \mathrm{~Hz}, 2 \mathrm{H}, \mathrm{Ph}-$ $\mathrm{H}), 2.46\left(\mathrm{~s}, 3 \mathrm{H}-\mathrm{CH}_{3}\right) ;{ }^{19} \mathrm{~F}$ NMR (471 MHz, chloroform-D) $\delta-62.42 ;{ }^{13} \mathrm{C}$ NMR (125 MHz, chloroform-D) $\delta$ 166.00, 161.15, $144.64(\mathrm{~d}, J=3.5 \mathrm{~Hz}), 144.14,139.24,136.74(\mathrm{q}, J=2.9 \mathrm{~Hz})$, 133.39, 132.15, 129.19, 128.71 (q, $J=34.1 \mathrm{~Hz}$ ), 128.03, 124.78, 123.11, 122.22 (q, $J=273.6 \mathrm{~Hz}), 21.43$; HR-MS (ESI): calculated for $\mathrm{C}_{15} \mathrm{H}_{9} \mathrm{ClF}_{3} \mathrm{~N}_{3} \mathrm{O}[\mathrm{M}+\mathrm{H}]^{+}: 340.04590$, found: 340.04696 .

2-(3-Chloro-5-(trifluoromethyl)pyridin-2-yl)-5-(3,5-dichlorophenyl)-1,3,4-oxadiazole (E5). Yield 32.0\%; light gray solid; mp 115.9-117.1 ${ }^{\circ} \mathrm{C} ;{ }^{1} \mathrm{H}$ NMR (500 MHz, chloroform-D) $\delta 8.97(\mathrm{~s}, 1 \mathrm{H}$, pyridine-H), 8.21 (d, $J=1.1 \mathrm{~Hz}, 1 \mathrm{H}$, pyridine- $\mathrm{H}), 8.10(\mathrm{~d}, J=$ $2.0 \mathrm{~Hz}, 2 \mathrm{H}, \mathrm{Ph}-\mathrm{H}), 7.58$ (t, $J=1.9 \mathrm{~Hz}, 1 \mathrm{H}, \mathrm{Ph}-\mathrm{H}) ;{ }^{19} \mathrm{~F}$ NMR (471 $\mathrm{MHz}$, chloroform-D) $\delta-62.46 ;{ }^{13} \mathrm{C}$ NMR $(125 \mathrm{MHz}$, chloroformD) $\delta 163.64,161.66,144.70(\mathrm{~d}, J=3.7 \mathrm{~Hz}), 143.66,136.83(\mathrm{~d}, J=$ $3.3 \mathrm{~Hz}$ ), 136.31, 132.47, 132.41, 129.09 (q, $J=34.5 \mathrm{~Hz}$ ), 125.89, 125.75, 122.13 (q, $J=273.6 \mathrm{~Hz}$ ); HR-MS (ESI): calculated for $\mathrm{C}_{14} \mathrm{H}_{5} \mathrm{Cl}_{3} \mathrm{~F}_{3} \mathrm{~N}_{3} \mathrm{O}[\mathrm{M}+\mathrm{H}]^{+}$: 393.95231, found: 393.95306.

2-(3-Chloro-5-(trifluoromethyl)pyridin-2-yl)-5-(4-ethylphenyl)1,3,4-oxadiazole (E6). Yield 66.9\%; yellow solid; mp 88.0-89.2 ${ }^{\circ} \mathrm{C}$; ${ }^{1} \mathrm{H}$ NMR (500 MHz, chloroform-D) $\delta 8.98-8.89(\mathrm{~m}, 1 \mathrm{H}$, pyridine$\mathrm{H}), 8.17(\mathrm{dd}, J=1.3,0.6 \mathrm{~Hz}, 1 \mathrm{H}$, pyridine- $\mathrm{H}), 8.10(\mathrm{~d}, J=8.2 \mathrm{~Hz}$, $2 \mathrm{H}, \mathrm{Ph}-\mathrm{H}), 7.36$ (d, $J=8.1 \mathrm{~Hz}, 2 \mathrm{H}, \mathrm{Ph}-\mathrm{H}), 2.73$ (q, $J=7.6 \mathrm{~Hz}, 2 \mathrm{H}$, $-\mathrm{CH}_{2}-$ ) 1.27 (t, $\left.J=7.6 \mathrm{~Hz}, 3 \mathrm{H},-\mathrm{CH}_{3}\right) ;{ }^{19} \mathrm{~F} \mathrm{NMR} \mathrm{(471} \mathrm{MHz,}$ chloroform-D) $\delta-62.44 ;{ }^{13} \mathrm{C}$ NMR (125 MHz, chloroform-D) $\delta$ 165.97, 160.99, 149.42, 144.59 (d, $J=3.6 \mathrm{~Hz}), 144.19,136.67$ $(\mathrm{d}, J=3.3 \mathrm{~Hz}), 132.08,128.78,128.63$ (q, $J=34.1 \mathrm{~Hz}), 127.64$, 122.23 (q, $J=273.4 \mathrm{~Hz}$ ), 120.67, 29.08, 15.23; HR-MS (ESI): calculated for $\mathrm{C}_{16} \mathrm{H}_{11} \mathrm{ClF}_{3} \mathrm{~N}_{3} \mathrm{O}[\mathrm{M}+\mathrm{H}]^{+}$: 354.06155 , found: 354.06137.

2-(3-Chloro-5-(trifluoromethyl)pyridin-2-yl)-5-(2,4-dimethyl phenyl)-1,3,4-oxadiazole (E7). Yield 63.8\%; white solid; mp 86.5-87.7 ${ }^{\circ} \mathrm{C} ;{ }^{1} \mathrm{H}$ NMR (500 MHz, chloroform-D) $\delta 8.95$ (dd, $J=$ $1.9,0.7 \mathrm{~Hz}, 1 \mathrm{H}$, pyridine-H), 8.19-8.16 ( $\mathrm{m}, 1 \mathrm{H}$, pyridine- $\mathrm{H}), 7.99$ (d, $J=7.9 \mathrm{~Hz}, 1 \mathrm{H}, \mathrm{Ph}-\mathrm{H}$ ), 7.16 (dd, $J=13.6,5.5 \mathrm{~Hz}, 3 \mathrm{H}-$ phenyl), $2.75\left(\mathrm{~s}, 3 \mathrm{H},-\mathrm{CH}_{3}\right), 2.40\left(\mathrm{~s}, 3 \mathrm{H},-\mathrm{CH}_{3}\right) ;{ }^{19} \mathrm{~F}$ NMR $(471 \mathrm{MHz}$, chloroform-D) $\delta-62.43 ;{ }^{13} \mathrm{C}$ NMR (125 MHz, chloroform-D) $\delta 166.25,160.60,144.63(\mathrm{~d}, J=3.9 \mathrm{~Hz}), 144.30,142.53,139.03$, $136.64(\mathrm{~d}, J=3.4 \mathrm{~Hz}), 132.76,132.07,129.56,128.59$ (q, $J=34.3$ $\mathrm{Hz}$ ), 127.18, 122.25 (q, $J=273.5 \mathrm{~Hz}$ ), 119.57, 22.21, 21.55; HRMS (ESI): calculated for $\mathrm{C}_{16} \mathrm{H}_{11} \mathrm{ClF}_{3} \mathrm{~N}_{3} \mathrm{O}[\mathrm{M}+\mathrm{H}]^{+}$: 354.06155, found: 354.06128 .

2-(2-Bromophenyl)-5-(3-chloro-5-(trifluoromethyl)pyridin-2yl)-1,3,4-oxadiazole (E8). Yield 46.8\%; light yellow solid; mp 75.7-77.0 ${ }^{\circ} \mathrm{C}$; ${ }^{1} \mathrm{H}$ NMR (500 MHz, chloroform-D) $\delta 8.96(\mathrm{~s}, 1 \mathrm{H}$, 
pyridine-H), $8.20(\mathrm{~s}, 1 \mathrm{H}$, pyridine- $\mathrm{H}), 8.05(\mathrm{dd}, J=7.8,1.3 \mathrm{~Hz}$, $1 \mathrm{H}, \mathrm{Ph}-\mathrm{H}), 7.79$ (d, $J=8.0 \mathrm{~Hz}, 1 \mathrm{H}, \mathrm{Ph}-\mathrm{H}), 7.50$ (dd, $J=11.8$, $4.4 \mathrm{~Hz}, 1 \mathrm{H}, \mathrm{Ph}-\mathrm{H}), 7.43$ (td, $J=7.8,1.4 \mathrm{~Hz}, 1 \mathrm{H}, \mathrm{Ph}-\mathrm{H}) ;{ }^{19} \mathrm{~F}$ NMR (471 MHz, chloroform-D) $\delta-62.44 ;{ }^{13} \mathrm{C}$ NMR $(125 \mathrm{MHz}$, chloroform-D) $\delta 164.74,161.66,144.75$ (d, $J=3.6 \mathrm{~Hz}), 144.01$, 136.75 (d, $J=3.3 \mathrm{~Hz}$ ), 134.78, 133.14, 132.35, 132.12, 128.92 (q, $J=34.3 \mathrm{~Hz}$ ), 127.79, 124.76, 122.19 (q, $J=273.5 \mathrm{~Hz}), 122.12$; HRMS (ESI): calculated for $\mathrm{C}_{14} \mathrm{H}_{6} \mathrm{ClF}_{4} \mathrm{~N}_{3} \mathrm{O}[\mathrm{M}+\mathrm{H}]^{+}$: 344.02083, found: 344.02029 .

2-(5-Bromo-2-chlorophenyl)-5-(3-chloro-5-(trifluoromethyl)pyridin-2-yl)-1,3,4-oxadiazole (E9). Yield 55.5\%; yellow solid; mp 99.4-100.6 ${ }^{\circ} \mathrm{C}$; ${ }^{1} \mathrm{H}$ NMR (500 MHz, chloroform-D) $\delta 8.96(\mathrm{~d}, J=$ $1.0 \mathrm{~Hz}, 1 \mathrm{H}$, pyridine- $\mathrm{H}), 8.26(\mathrm{~d}, J=2.3 \mathrm{~Hz}, 1 \mathrm{H}$, pyridine- $\mathrm{H}), 8.20$ (d, $J=1.6 \mathrm{~Hz}, 1 \mathrm{H}, \mathrm{Ph}-\mathrm{H}), 7.62$ (dd, $J=8.6,2.5 \mathrm{~Hz}, 1 \mathrm{H}, \mathrm{Ph}-\mathrm{H}$ ), 7.45 (d, $J=8.6 \mathrm{~Hz}, 1 \mathrm{H}, \mathrm{Ph}-\mathrm{H}) ;{ }^{19} \mathrm{~F}$ NMR $(471 \mathrm{MHz}$, chloroform-D) $\delta-62.44 ;{ }^{13} \mathrm{C}$ NMR (125 MHz, chloroform-D) $\delta 162.99,161.77,144.78(\mathrm{~d}, J=3.4 \mathrm{~Hz}), 143.79,136.80(\mathrm{~d}, J=$ $2.9 \mathrm{~Hz}), 135.97,134.05,132.88,132.64,132.47,129.07$ (q, $J=$ $34.1 \mathrm{~Hz}), 124.16,122.15$ (q, $J=273.7 \mathrm{~Hz}$ ), 120.86; HR-MS (ESI): calculated for $\mathrm{C}_{14} \mathrm{H}_{5} \mathrm{BrCl}_{2} \mathrm{~F}_{3} \mathrm{~N}_{3} \mathrm{O}[\mathrm{M}+\mathrm{H}]^{+}$: 437.90179, found: 437.90149.

2-(3-Chloro-5-(trifluoromethyl)pyridin-2-yl)-5-(4-iodophenyl)1,3,4-oxadiazole (E10). Yield 48.0\%; yellow solid; mp 153.0$153.7{ }^{\circ} \mathrm{C}$; ${ }^{1} \mathrm{H}$ NMR $(500 \mathrm{MHz}$, chloroform-D) $\delta 8.95(\mathrm{~s}, 1 \mathrm{H}$, pyridine-H), 8.20 (s, 1H, pyridine- $\mathrm{H}), 7.92(\mathrm{~d}, J=0.5 \mathrm{~Hz}, 4 \mathrm{H}, \mathrm{Ph}-$ $\mathrm{H}) ;{ }^{19} \mathrm{~F}$ NMR (471 MHz, chloroform-D) $\delta-62.44 ;{ }^{13} \mathrm{C}$ NMR $(125$ MHz, chloroform-D) $\delta 165.29,161.33,144.66$ (d, $J=3.5 \mathrm{~Hz}$ ), $143.89,138.62,136.80(\mathrm{~d}, J=3.3 \mathrm{~Hz}), 132.28,128.87(\mathrm{q}, J=34.3$ $\mathrm{Hz}), 128.83,122.67,122.17$ (q, $J=273.5 \mathrm{~Hz}$ ), 99.76; HR-MS (ESI): calculated for $\mathrm{C}_{14} \mathrm{H}_{6} \mathrm{ClF}_{3} \mathrm{IN}_{3} \mathrm{O}[\mathrm{M}+\mathrm{H}]^{+}$: 451.92689, found: 451.92648.

2-(4-Bromophenyl)-5-(3-chloro-5-(trifluoromethyl)pyridin-2-yl)1,3,4-oxadiazole (E11). Yield 37.9\%; light yellow solid; mp 151.2$154.9{ }^{\circ} \mathrm{C} ;{ }^{1} \mathrm{H}$ NMR (500 MHz, chloroform-D) $\delta 8.95(\mathrm{~d}, J=0.6 \mathrm{~Hz}$, $1 \mathrm{H}$, pyridine- $\mathrm{H}), 8.21-8.18(\mathrm{~m}, 1 \mathrm{H}$, pyridine- $\mathrm{H}), 8.08(\mathrm{dd}, J=8.3$, $0.5 \mathrm{~Hz}, 2 \mathrm{H}, \mathrm{Ph}-\mathrm{H}$ ), 7.70 (dd, $J=7.1,1.2 \mathrm{~Hz}, 2 \mathrm{H}, \mathrm{Ph}-\mathrm{H}) ;{ }^{19} \mathrm{~F}$ NMR (471 MHz, chloroform-D) $\delta-62.45 ;{ }^{13} \mathrm{C}$ NMR $(125 \mathrm{MHz}$, chloroform-D) $\delta$ 165.08, 161.31, 144.63 (d, $J=3.5 \mathrm{~Hz}$ ), 143.91, 136.75 (d, $J=3.3 \mathrm{~Hz}), 132.66,132.26,128.92,128.81$ (q, $J=27.1$ $\mathrm{Hz}), 127.40,122.18$ (q, $J=273.7 \mathrm{~Hz}$ ), 122.17; HR-MS (ESI): calculated for $\mathrm{C}_{14} \mathrm{H}_{6} \mathrm{BrClF}_{3} \mathrm{~N}_{3} \mathrm{O}[\mathrm{M}+\mathrm{H}]^{+}$: 403.94076, found: 403.94077.

2-(4-Bromo-2-fluorophenyl)-5-(3-chloro-5-(trifluoromethyl)pyridin-2-yl)-1,3,4-oxadiazole (E12). Yield 10.9\%; light yellow solid; mp 105.9-107.1 ${ }^{\circ} \mathrm{C} ;{ }^{1} \mathrm{H}$ NMR (500 MHz, chloroform-D) $\delta 8.97(\mathrm{t}, J=5.9 \mathrm{~Hz}, 1 \mathrm{H}$, pyridine- $\mathrm{H}), 8.21(\mathrm{t}, J=5.7 \mathrm{~Hz}, 1 \mathrm{H}$, pyridine-H), 8.09 (t, $J=7.8 \mathrm{~Hz}, 1 \mathrm{H}, \mathrm{Ph}-\mathrm{H}), 7.51$ (d, $J=8.6 \mathrm{~Hz}$, $2 \mathrm{H}, \mathrm{Ph}-\mathrm{H}) ;{ }^{19} \mathrm{~F}$ NMR (471 MHz, chloroform-D) $\delta-62.47$, $-106.35 ;{ }^{13} \mathrm{C}$ NMR (125 MHz, chloroform-D) $\delta 162.04(\mathrm{~d}, J=4.8$ $\mathrm{Hz}), 161.57,159.97$ (d, $J=264.2 \mathrm{~Hz}), 144.75$ (d, $J=3.7 \mathrm{~Hz}$ ), 143.86, 136.75 (d, $J=3.2 \mathrm{~Hz}), 132.41,130.99,128.99$ (d, $J=34.1$ $\mathrm{Hz}), 128.55$ (d, $J=3.5 \mathrm{~Hz}), 127.75$ (d, $J=9.3 \mathrm{~Hz}), 124.34$ (d, $J=$ $274.4 \mathrm{~Hz}), 121.01$ (d, $J=23.8 \mathrm{~Hz}), 113.43,111.02(\mathrm{~d}, J=11.4 \mathrm{~Hz})$; HR-MS (ESI): calculated for $\mathrm{C}_{14} \mathrm{H}_{5} \mathrm{BrClF}_{4} \mathrm{~N}_{3} \mathrm{O}[\mathrm{M}+\mathrm{H}]^{+}$: 421.93134, found: 421.93088 .

2-(3-Chloro-5-(trifluoromethyl)pyridin-2-yl)-5-(2-fluorophenyl)1,3,4-oxadiazole (E13). Yield 34.1\%; light red solid; mp 83.6-
$84.8{ }^{\circ} \mathrm{C} ;{ }^{1} \mathrm{H}$ NMR $(500 \mathrm{MHz}$, chloroform-D) $\delta 8.96(\mathrm{~d}, J=1.0 \mathrm{~Hz}$, $1 \mathrm{H}$, pyridine- $\mathrm{H}), 8.21$ (dd, $J=7.5,1.5 \mathrm{~Hz}, 1 \mathrm{H}$, pyridine- $\mathrm{H}), 8.19$ (d, $J=1.8 \mathrm{~Hz}, 1 \mathrm{H}, \mathrm{Ph}-\mathrm{H}), 7.63-7.55$ (m, 1H, Ph-H), 7.37-7.27 (m, 2H, Ph-H); ${ }^{19}$ F NMR (471 MHz, chloroform-D) $\delta-62.47,-108.87 ;{ }^{13} \mathrm{C}$ NMR (125 MHz, chloroform-D) $\delta 162.66$ (d, $J=4.8 \mathrm{~Hz}), 161.44$, $160.42(\mathrm{~d}, J=262.9 \mathrm{~Hz}), 144.72(\mathrm{~d}, J=3.6 \mathrm{~Hz}), 144.03,136.70(\mathrm{~d}, J$ $=3.3 \mathrm{~Hz}), 134.34(\mathrm{~d}, J=8.5 \mathrm{~Hz}), 132.32,130.27,128.87$ (q, $J=34.0$ $\mathrm{Hz}), 124.89$ (d, $J=3.5 \mathrm{~Hz}), 122.19$ (q, $J=273.5 \mathrm{~Hz}), 117.21$ (d, $J=$ $20.7 \mathrm{~Hz}$ ), 111.88 (d, $J=11.3 \mathrm{~Hz}$ ); HR-MS (ESI): calculated for $\mathrm{C}_{14} \mathrm{H}_{6} \mathrm{ClF}_{4} \mathrm{~N}_{3} \mathrm{O}[\mathrm{M}+\mathrm{H}]^{+}: 344.02083$, found: 344.02042 .

2-(3-Bromophenyl)-5-(3-chloro-5-(trifluoromethyl)pyridin-2-yl)1,3,4-oxadiazole (E14). Yield 42.4\%; light gray solid; mp 107.8$108.9{ }^{\circ} \mathrm{C} ;{ }^{1} \mathrm{H}$ NMR (500 MHz, chloroform-D) $\delta 8.96(\mathrm{~d}, J=1.1 \mathrm{~Hz}$, $1 \mathrm{H}$, pyridine- $\mathrm{H}), 8.35(\mathrm{t}, J=1.7 \mathrm{~Hz}, 1 \mathrm{H}$, pyridine- $\mathrm{H}), 8.22-8.19(\mathrm{~m}$, 1H, Ph-H), 8.17-8.14 (m, 1H, Ph-H), 7.73 (ddd, $J=8.0,1.8,1.0 \mathrm{~Hz}$, $1 \mathrm{H}, \mathrm{Ph}-\mathrm{H}), 7.44$ (t, $J=7.9 \mathrm{~Hz}, 1 \mathrm{H}, \mathrm{Ph}-\mathrm{H}) ;{ }^{19} \mathrm{~F}$ NMR $(471 \mathrm{MHz}$, chloroform-D) $\delta-62.44 ;{ }^{13} \mathrm{C}$ NMR (125 MHz, chloroform-D) $\delta 164.50,161.44,144.67(\mathrm{~d}, J=3.8 \mathrm{~Hz}), 143.86,136.78(\mathrm{~d}, J=$ $3.4 \mathrm{~Hz}), 135.51,132.33,130.84,130.32,128.92$ (q, $J=34.4 \mathrm{~Hz}$ ), 126.11, 125.09, 123.33, 122.17 (q, $J=273.4 \mathrm{~Hz}$ ); HR-MS (ESI): calculated for $\mathrm{C}_{14} \mathrm{H}_{6} \mathrm{BrClF}_{3} \mathrm{~N}_{3} \mathrm{O}[\mathrm{M}+\mathrm{H}]^{+}$: 403.94076, found: 403.94049.

2-(3-Chloro-5-(trifluoromethyl)pyridin-2-yl)-5-(3,4-dichlorophenyl)-1,3,4-oxadiazole (E15). Yield 36.6\%; gray solid; mp 142.6-143.7 ${ }^{\circ} \mathrm{C} ;{ }^{1} \mathrm{H}$ NMR (500 MHz, chloroform-D) $\delta$ 8.97-8.93 ( $\mathrm{m}, 1 \mathrm{H}$, pyridine $\mathrm{H}), 8.29(\mathrm{~d}, J=2.0 \mathrm{~Hz}, 1 \mathrm{H}$, pyridine- $\mathrm{H}), 8.21$ (d, $J=1.3 \mathrm{~Hz}, 1 \mathrm{H}, \mathrm{Ph}-\mathrm{H}), 8.05$ (dd, $J=8.4,2.0 \mathrm{~Hz}, 1 \mathrm{H}, \mathrm{Ph}-\mathrm{H}$ ), 7.64 (d, $J=8.4 \mathrm{~Hz}, 1 \mathrm{H}, \mathrm{Ph}-\mathrm{H}) ;{ }^{19} \mathrm{~F}$ NMR (471 MHz, chloroformD) $\delta-62.43 ;{ }^{13} \mathrm{C}$ NMR $(125 \mathrm{MHz}$, chloroform-D) $\delta$ 164.02, 161.52, 144.68, 143.69, 137.20, 136.85, 134.03, 132.40, 131.53, 129.20, 129.00 (q, $J=273.5 \mathrm{~Hz}$ ), 126.55, 123.00, 121.05; HR-MS (ESI): calculated for $\mathrm{C}_{14} \mathrm{H}_{5} \mathrm{Cl}_{3} \mathrm{~F}_{3} \mathrm{~N}_{3} \mathrm{O}[\mathrm{M}+\mathrm{H}]^{+}$: 393.95231, found: 393.95209 .

2-(3-Chloro-5-(trifluoromethyl)pyridin-2-yl)-5-(4-chlorophenyl)1,3,4-oxadiazole (E16). Yield 17.5\%; white solid; mp 169.8$170.3{ }^{\circ} \mathrm{C} ;{ }^{1} \mathrm{H}$ NMR (500 MHz, chloroform-D) $\delta 8.94(\mathrm{~s}, 1 \mathrm{H}$, pyridine-H), 8.19 (s, 1H, pyridine-H), $8.13(\mathrm{~d}, J=8.4 \mathrm{~Hz}, 2 \mathrm{H}, \mathrm{Ph}-$ $\mathrm{H}), 7.52(\mathrm{~d}, J=8.4 \mathrm{~Hz}, 2 \mathrm{H}, \mathrm{Ph}-\mathrm{H}) ;{ }^{13} \mathrm{C}$ NMR $(125 \mathrm{MHz}$, chloroform-D) $\delta$ 164.99, 161.29, 144.64 (d, $J=3.6 \mathrm{~Hz}), 143.90$, $138.93,136.77$ (d, $J=3.1 \mathrm{~Hz}), 132.25,129.70,128.84$ (q, $J=34.0$ $\mathrm{Hz}), 128.82,122.17$ (d, $J=273.4 \mathrm{~Hz}$ ), 121.72; HR-MS (ESI): calculated for $\mathrm{C}_{14} \mathrm{H}_{6} \mathrm{Cl}_{2} \mathrm{~F}_{3} \mathrm{~N}_{3} \mathrm{O}[\mathrm{M}+\mathrm{H}]^{+}$: 359.99128, found: 359.99078.

2-(3-Chloro-5-(trifluoromethyl)pyridin-2-yl)-5-(3-chlorophenyl)1,3,4-oxadiazole (E17). Yield 40.6\%; pink solid; mp 93.6-94.8 ${ }^{\circ} \mathrm{C}$; ${ }^{1} \mathrm{H}$ NMR (500 MHz, chloroform-D) $\delta 8.96(\mathrm{~s}, 1 \mathrm{H}$, pyridine-H), 8.20 (d, $J=0.9 \mathrm{~Hz}, 1 \mathrm{H}$, pyridine-H), 8.20-8.18 (m, 1H, Ph-H), 8.13-8.08 (m, 1H, Ph-H), 7.58-7.55 (m, 1H, Ph-H), 7.50 (t, $J=7.9 \mathrm{~Hz}, 1 \mathrm{H}, \mathrm{Ph}-$ $\mathrm{H}) ;{ }^{19} \mathrm{~F}$ NMR (471 MHz, chloroform-D) $\delta-62.43 ;{ }^{13} \mathrm{C}$ NMR (125 $\mathrm{MHz}$, chloroform-D) $\delta 164.67,161.45,144.67,143.85,136.80$, 135.49, 132.61, 132.33, 130.67, 128.93 (q, $J=33.9 \mathrm{~Hz}$ ), 127.48, 125.69, 124.87, 122.16 (q, $J=273.5 \mathrm{~Hz}$ ); HR-MS (ESI): calculated for $\mathrm{C}_{14} \mathrm{H}_{6} \mathrm{Cl}_{2} \mathrm{~F}_{3} \mathrm{~N}_{3} \mathrm{O}[\mathrm{M}+\mathrm{H}]^{+}: 359.99128$, found: 359.99078 .

2-(3-Chloro-5-(trifluoromethyl)pyridin-2-yl)-5-(2-(trifluoromethyl)phenyl)-1,3,4-oxadiazole (E18). Yield 55.0\%; yellow solid; mp 60.8-61.5 ${ }^{\circ} \mathrm{C} ;{ }^{1} \mathrm{H}$ NMR (500 MHz, chloroform-D) $\delta 8.96$ (s, $1 \mathrm{H}$, pyridine- $\mathrm{H}), 8.20(\mathrm{~s}, 1 \mathrm{H}$, pyridine $-\mathrm{H}), 8.15-8.12(\mathrm{~m}, 1 \mathrm{H}$, 
Ph-H), 7.93-7.88 (m, 1H, Ph-H), 7.77-7.74 (m, 2H, Ph-H); ${ }^{19} \mathrm{~F}$ NMR (471 MHz, chloroform-D) $\delta-59.71,-62.52 ;{ }^{13} \mathrm{C}$ NMR (125 MHz, chloroform-D) $\delta 164.14,162.29,144.77$ (d, $J=3.9 \mathrm{~Hz}$ ), 143.91, 136.71 (d, $J=3.4 \mathrm{~Hz}), 132.40,132.31,132.22,129.38$ (q, $J$ $=32.6 \mathrm{~Hz}), 129.02(\mathrm{q}, J=33.9 \mathrm{~Hz}), 127.26(\mathrm{q}, J=5.0 \mathrm{~Hz}), 123.17$ (q, $J=273.6 \mathrm{~Hz}), 121.74,122.17$ (q, $J=273.5 \mathrm{~Hz}$ ); HR-MS (ESI): calculated for $\mathrm{C}_{15} \mathrm{H}_{6} \mathrm{ClF}_{6} \mathrm{~N}_{3} \mathrm{O}[\mathrm{M}+\mathrm{H}]^{+}$: 394.01764, found: 394.01730.

2-(3-Chloro-5-(trifluoromethyl)pyridin-2-yl)-5-(4-(trifluoromethyl)phenyl)-1,3,4-oxadiazole (E19). Yield 45.0\%; light yellow solid; mp 109.9-111.0 ${ }^{\circ} \mathrm{C}$; ${ }^{1} \mathrm{H}$ NMR (500 MHz, chloroform-D) $\delta 8.97(\mathrm{~s}, 1 \mathrm{H}$, pyridine-H), $8.35(\mathrm{~s}, 1 \mathrm{H}$, pyridine- $\mathrm{H}), 8.34(\mathrm{~s}, 1 \mathrm{H}$, Ph-H), 8.21 (s, 1H, Ph-H), 7.83 (d, $J=8.2 \mathrm{~Hz}, 2 \mathrm{H}, \mathrm{Ph}-\mathrm{H}) ;{ }^{19} \mathrm{~F}$ NMR (471 MHz, chloroform-D) $\delta-62.47,-63.10 ;{ }^{13} \mathrm{C} \mathrm{NMR}(125 \mathrm{MHz}$, chloroform-D) $\delta 164.59,161.58, \delta 144.69(\mathrm{~d}, J=3.7 \mathrm{~Hz}), 143.79$, $\delta 136.81(\mathrm{~d}, J=3.4 \mathrm{~Hz}), \delta 134.09(\mathrm{q}, J=33.2 \mathrm{~Hz}), 132.42, \delta 129.02$ $(\mathrm{q}, J=34.4 \mathrm{~Hz}), 127.92,126.51, \delta 126.33(\mathrm{~d}, J=3.6 \mathrm{~Hz}), \delta 123.55$ (q, $J=272.6 \mathrm{~Hz}$ ), $\delta 122.15$ (q, $J=273.5 \mathrm{~Hz}$ ); HR-MS (ESI): calculated for $\mathrm{C}_{15} \mathrm{H}_{6} \mathrm{ClF}_{6} \mathrm{~N}_{3} \mathrm{O}[\mathrm{M}+\mathrm{H}]^{+}$: 394.01764, found: 394.01740 .

2-(3-Chloro-5-(trifluoromethyl)pyridin-2-yl)-5-(2-methoxyphenyl)-1,3,4-oxadiazole (E20). Yield 70.3\%; purple solid; mp 67.0-68.2 ${ }^{\circ} \mathrm{C} ;{ }^{1} \mathrm{H}$ NMR (500 MHz, chloroform-D) $\delta 8.95(\mathrm{~d}, J=$ $0.7 \mathrm{~Hz}, 1 \mathrm{H}$, pyridine- $\mathrm{H}), 8.18(\mathrm{~s}, 1 \mathrm{H}$, pyridine- $\mathrm{H}), 8.07(\mathrm{~d}, J=$ $6.6 \mathrm{~Hz}, 1 \mathrm{H}, \mathrm{Ph}-\mathrm{H}), 7.57-7.52(\mathrm{~m}, 1 \mathrm{H}, \mathrm{Ph}-\mathrm{H}), 7.09$ (dd, $J=14.3$, $7.8 \mathrm{~Hz}, 2 \mathrm{H}, \mathrm{Ph}-\mathrm{H}), 3.99\left(\mathrm{~s}, 3 \mathrm{H}-\mathrm{CH}_{3}\right) ;{ }^{19} \mathrm{~F}$ NMR $(471 \mathrm{MHz}$, chloroform-D) $\delta-62.42 ;{ }^{13} \mathrm{C}$ NMR (125 MHz, chloroform-D) $\delta 164.70,161.01,158.34,144.63,144.36,136.65,133.88$, 132.11, 131.01, 128.60 (q, $J=34.5 \mathrm{~Hz}), 122.25(\mathrm{q}, J=272.8 \mathrm{~Hz})$, 120.92, 112.36, 112.08, 56.17; HR-MS (ESI): calculated for $\mathrm{C}_{15^{-}}$ $\mathrm{H}_{9} \mathrm{ClF}_{3} \mathrm{~N}_{3} \mathrm{O}_{2}[\mathrm{M}+\mathrm{H}]^{+}$: 355.03299, found: 355.03272.

2-(4-Chloro-3-fluorophenyl)-5-(3-chloro-5-(trifluoromethyl)pyridin-2-yl)-1,3,4-oxadiazole (E21). Yield 23.9\%; light purple solid; mp 140.7-141.7 ${ }^{\circ} \mathrm{C} ;{ }^{1} \mathrm{H}$ NMR (500 MHz, chloroform-D) $\delta 8.96(\mathrm{~s}, 1 \mathrm{H}$, pyridine-H), 8.21 (s, 1H, pyridine- $\mathrm{H}), 8.02-7.95$ $(\mathrm{m}, 2 \mathrm{H}, \mathrm{Ph}-\mathrm{H}), 7.61$ (t, $J=7.7 \mathrm{~Hz}, 1 \mathrm{H}, \mathrm{Ph}-\mathrm{H}) ;{ }^{19} \mathrm{~F} \mathrm{NMR}(471 \mathrm{MHz}$, chloroform-D) $\delta-62.46,-112.35 ;{ }^{13} \mathrm{C}$ NMR $(125 \mathrm{MHz}$, chloroform-D) $\delta 164.10(\mathrm{~d}, J=2.1 \mathrm{~Hz}), 161.50,158.45(\mathrm{~d}, J=$ $251.2 \mathrm{~Hz}), 144.68$ (d, $J=3.6 \mathrm{~Hz}), 143.73,136.81(\mathrm{~d}, J=3.4 \mathrm{~Hz})$, 132.38, 131.88, 129.00 (q, $J=34.4 \mathrm{~Hz}), 125.93$ (d, $J=17.8 \mathrm{~Hz}$ ), $123.91(\mathrm{~d}, J=3.7 \mathrm{~Hz}), 123.39$ (d, $J=7.5 \mathrm{~Hz}), 122.15$ (q, $J=273.6$ $\mathrm{Hz}), 115.64$ (d, $J=24.1 \mathrm{~Hz}$ ); HR-MS (ESI): calculated for $\mathrm{C}_{14^{-}}$ $\mathrm{H}_{5} \mathrm{Cl}_{2} \mathrm{~F}_{4} \mathrm{~N}_{3} \mathrm{O}[\mathrm{M}+\mathrm{H}]^{+}$: 377.98186 , found: 377.98135 .

2-(3-Chloro-5-(trifluoromethyl)pyridin-2-yl)-5-(4-fluorophenyl)1,3,4-oxadiazole (E22). Yield 52.5\%; light red solid; mp 99.1$100.4{ }^{\circ} \mathrm{C} ;{ }^{1} \mathrm{H}$ NMR (500 MHz, chloroform-D) $\delta 8.94(\mathrm{~d}, J=1.8 \mathrm{~Hz}$, $1 \mathrm{H}$, pyridine- $\mathrm{H}), 8.23-8.22(\mathrm{~m}, 1 \mathrm{H}$, pyridine- $\mathrm{H}), 8.21-8.18(\mathrm{~m}, 2 \mathrm{H}$, Ph-H), 7.26-7.21 (m, 2H, Ph-H); ${ }^{19}$ F NMR (471 MHz, chloroformD) $\delta-62.43,-105.33 .{ }^{13} \mathrm{C}$ NMR $(125 \mathrm{MHz}$, chloroform-D, ppm) $\delta 165.33(\mathrm{~d}, J=254.4 \mathrm{~Hz}), 165.00,161.22,144.64(\mathrm{~d}, J=3.4 \mathrm{~Hz})$, 143.96, 136.77 (d, $J=3.4 \mathrm{~Hz}), 132.20,129.96(\mathrm{~d}, J=8.9 \mathrm{~Hz})$, $128.80(\mathrm{q}, J=34.1 \mathrm{~Hz}), 122.18(\mathrm{q}, J=273.4 \mathrm{~Hz}), 119.57,116.71(\mathrm{~d}$, $J=22.2 \mathrm{~Hz}$ ); HR-MS (ESI): calculated for $\mathrm{C}_{14} \mathrm{H}_{6} \mathrm{ClF}_{4} \mathrm{~N}_{3} \mathrm{O}[\mathrm{M}+\mathrm{H}]^{+}$: 344.02083, found: 344.02029 .

2-(3-Chloro-5-(trifluoromethyl)pyridin-2-yl)-5-(3-(trifluoromethyl)phenyl)-1,3,4-oxadiazole (E23). Yield 45.8\%; yellow solid; mp 81.9-83.0 ${ }^{\circ} \mathrm{C} ;{ }^{1} \mathrm{H}$ NMR (500 MHz, chloroform-D) $\delta 8.97$ (dd, $J=1.8,0.7 \mathrm{~Hz}, 1 \mathrm{H}$, pyridine-H), 8.47 (d, $J=0.6 \mathrm{~Hz}, 1 \mathrm{H}$, pyridine-H), 8.42 (d, $J=7.8 \mathrm{~Hz}, 1 \mathrm{H}, \mathrm{Ph}-\mathrm{H}), 8.22$ (dd, $J=1.2$, $0.7 \mathrm{~Hz}, 1 \mathrm{H}, \mathrm{Ph}-\mathrm{H}$ ), 7.86 (dd, $J=7.8,0.7 \mathrm{~Hz}, 1 \mathrm{H}, \mathrm{Ph}-\mathrm{H}), 7.72$ (t, $J=$ $7.8 \mathrm{~Hz}, 1 \mathrm{H}, \mathrm{Ph}-\mathrm{H}) ;{ }^{19} \mathrm{~F}$ NMR (471 MHz, chloroform-D) $\delta-62.49$, $-62.88 ;{ }^{13} \mathrm{C}$ NMR (125 MHz, chloroform-D) $\delta 164.57,161.55$, $144.68(\mathrm{~d}, J=3.6 \mathrm{~Hz}), 143.80,136.79$ (d, $J=3.4 \mathrm{~Hz}), 132.40$, $132.04(\mathrm{~d}, J=33.2 \mathrm{~Hz}), 130.66,130.01,129.02(\mathrm{~d}, J=3.4 \mathrm{~Hz})$, $129.00(\mathrm{q}, J=33.9 \mathrm{~Hz}), 123.50(\mathrm{q}, J=272.7 \mathrm{~Hz}), 124.39$ (d, $J=3.7$ $\mathrm{Hz}), 124.16,122.15$ (q, $J=273.5 \mathrm{~Hz}$ ). HR-MS (ESI): calculated for $\mathrm{C}_{15} \mathrm{H}_{6} \mathrm{ClF}_{6} \mathrm{~N}_{3} \mathrm{O}[\mathrm{M}+\mathrm{H}]^{+}$: 394.01764, found: 394.01685 .

2-(3-Chloro-5-(trifluoromethyl)pyridin-2-yl)-5-(3-iodophenyl)1,3,4-oxadiazole (E24). Yield 45.0\%; light yellow solid; $\mathrm{mp}$ 121.4-123. $6{ }^{\circ} \mathrm{C} ;{ }^{1} \mathrm{H}$ NMR (500 MHz, chloroform-D) $\delta 8.96(\mathrm{dd}, J=$ $1.7,0.6 \mathrm{~Hz}, 1 \mathrm{H}$, pyridine- $\mathrm{H}), 8.53(\mathrm{t}, J=1.6 \mathrm{~Hz}, 1 \mathrm{H}$, pyridine- $\mathrm{H})$, 8.21-8.16 (m, 2H, Ph-H), 7.92 (ddd, $J=7.9,1.7,1.1 \mathrm{~Hz}, 1 \mathrm{H}, \mathrm{Ph}-$ $\mathrm{H}), 7.29(\mathrm{t}, J=7.9 \mathrm{~Hz}, 1 \mathrm{H}, \mathrm{Ph}-\mathrm{H}) ;{ }^{19} \mathrm{~F} \mathrm{NMR}(471 \mathrm{MHz}$, chloroform-D) $\delta-62.43 ;{ }^{13} \mathrm{C}$ NMR (125 MHz, chloroform-D) $\delta$ 164.32, 161.43, 144.68 (d, $J=3.6 \mathrm{~Hz}), 143.86,141.44,136.81$ (d, $J=3.3 \mathrm{~Hz}), 136.05,132.33,130.88,128.92(\mathrm{q}, J=34.4 \mathrm{~Hz})$, 126.70, 125.06, 122.17 (d, $J=273.5 \mathrm{~Hz}$ ), 94.56; HR-MS (ESI): calculated for $\mathrm{C}_{14} \mathrm{H}_{6} \mathrm{ClF}_{3} \mathrm{IN}_{3} \mathrm{O}[\mathrm{M}+\mathrm{H}]^{+}$: 451.92689, found: 451.92661.

2-(3-Chloro-5-(trifluoromethyl)pyridin-2-yl)-5-(2-iodophenyl)1,3,4-oxadiazole (E25). Yield 66.0\%; yellow solid; mp 54.4$55.4{ }^{\circ} \mathrm{C} ;{ }^{1} \mathrm{H}$ NMR (500 MHz, chloroform-D) $\delta 8.95$ (dd, $J=1.7$, $0.7 \mathrm{~Hz}, 1 \mathrm{H}$, pyridine-H), 8.21-8.18 (m, 1H, pyridine-H), 8.08 (dd, $J=8.0,1.1 \mathrm{~Hz}, 1 \mathrm{H}, \mathrm{Ph}-\mathrm{H}), 7.94$ (dd, $J=7.8,1.7 \mathrm{~Hz}, 1 \mathrm{H}, \mathrm{Ph}-\mathrm{H}$ ), 7.52 (td, $J=7.6,1.2 \mathrm{~Hz}, 1 \mathrm{H}, \mathrm{Ph}-\mathrm{H}), 7.26-7.23$ (m, 1H, Ph-H); ${ }^{19} \mathrm{~F}$ NMR (471 MHz, chloroform-D) $\delta-62.43 ;{ }^{13} \mathrm{C}$ NMR (125 $\mathrm{MHz}$, chloroform-D) $\delta 165.43,161.61,144.74,144.71,143.98$, $141.59,136.79,130.81,132.36,131.87,129.04,128.92$ (q, $J=34.4$ $\mathrm{Hz}$ ), 128.44, 122.17 (q, $J=273.6 \mathrm{~Hz}$ ). 94.60; HR-MS (ESI): calculated for $\mathrm{C}_{14} \mathrm{H}_{6} \mathrm{ClF}_{3} \mathrm{IN}_{3} \mathrm{O}[\mathrm{M}+\mathrm{H}]^{+}:$451.92689, found: 451.92636.

3-(5-(3-Chloro-5-(trifluoromethyl)pyridin-2-yl)-1,3,4-oxadiazol2-yl)- $\mathbf{N}, \boldsymbol{N}$-dimethylaniline (E26). Yield 29.4\%; yellow solid; mp 76.5-78.6 ${ }^{\circ} \mathrm{C} ;{ }^{1} \mathrm{H}$ NMR (500 MHz, chloroform-D) $\delta 8.95$ (dd, $J=$ 1.8, $0.7 \mathrm{~Hz}, 1 \mathrm{H}$, pyridine-H), 8.18 (dd, $J=1.3,0.6 \mathrm{~Hz}, 1 \mathrm{H}$, pyridine-H), 7.54-7.47 (m, 2H, Ph-H), 7.40-7.35 (m, 1H, Ph-H), 6.94-6.89 (m, 1H, Ph-H), $3.04\left(\mathrm{~s}, 6 \mathrm{H},-\mathrm{CH}_{3}\right) ;{ }^{19} \mathrm{~F}$ NMR (471 $\mathrm{MHz}$, chloroform-D) $\delta-62.43 ;{ }^{13} \mathrm{C}$ NMR (125 MHz, chloroformD) $\delta 166.62,161.05,150.81,144.61(\mathrm{~d}, J=3.5 \mathrm{~Hz}), 144.31$, $136.64(\mathrm{~d}, J=3.2 \mathrm{~Hz}), 132.09,129.92,128.65(\mathrm{q}, J=33.9 \mathrm{~Hz})$, 123.80, 122.25 (d, $J=273.5 \mathrm{~Hz}$ ), 116.28, 115.40, 110.62, 40.53; HR-MS (ESI): calculated for $\mathrm{C}_{16} \mathrm{H}_{12} \mathrm{ClF}_{3} \mathrm{~N}_{4} \mathrm{O}[\mathrm{M}+\mathrm{H}]^{+}:$369.07246, found: 369.07220 .

2-(3-Chloro-5-(trifluoromethyl)pyridin-2-yl)-5-(4-fluorobenzyl)1,3,4-oxadiazole (E27). Yield 53.2\%; yellow solid; mp 90.1$91.4{ }^{\circ} \mathrm{C} ;{ }^{1} \mathrm{H}$ NMR $(500 \mathrm{MHz}$, chloroform-D) $\delta 8.89$ (s, $1 \mathrm{H}$, pyridine$\mathrm{H}), 8.15$ (s, 1H, pyridine-H), 7.35 (dd, $J=8.1,5.4 \mathrm{~Hz}, 2 \mathrm{H}, \mathrm{Ph}-\mathrm{H}$ ), 7.04 (t, $J=8.4 \mathrm{~Hz}, 2 \mathrm{H}, \mathrm{Ph}-\mathrm{H}), 4.33\left(\mathrm{~s}, 2 \mathrm{H},-\mathrm{CH}_{2}-\right) ;{ }^{13} \mathrm{C}$ NMR (125 MHz, chloroform-D) $\delta 166.59(\mathrm{~d}, J=1.2 \mathrm{~Hz}), 161.79,162.41(\mathrm{~d}, J=$ $246.6 \mathrm{~Hz}), 144.57$ (q, $J=3.8 \mathrm{~Hz}), 136.75$ (q, $J=3.8 \mathrm{~Hz}), 132.23$, 130.71, 130.64, 129.03 (d, $J=3.3 \mathrm{~Hz}), 128.86$ (d, $J=34.3 \mathrm{~Hz}$ ), $122.13(\mathrm{~d}, J=273.5 \mathrm{~Hz}$ ), 116.04 (d, $J=21.7 \mathrm{~Hz}$ ), 31.15. HR-MS (ESI): calculated for $\mathrm{C}_{15} \mathrm{H}_{8} \mathrm{ClF}_{4} \mathrm{~N}_{3} \mathrm{O}[\mathrm{M}+\mathrm{H}]^{+}$: 358.03648, found: 358.03482 . 
2-(3-Chloro-5-(trifluoromethyl)pyridin-2-yl)-5-(2,4-dichlorophenyl)-1,3,4-oxadiazole (E28). Yield 50.3\%; gray solid; mp 80.0-83.8 ${ }^{\circ} \mathrm{C} ;{ }^{1} \mathrm{H}$ NMR (500 MHz, chloroform-D) $\delta 8.96(\mathrm{~d}, J=$ $0.9 \mathrm{~Hz}, 1 \mathrm{H}$, pyridine- $\mathrm{H}), 8.20(\mathrm{~d}, J=1.1 \mathrm{~Hz}, 1 \mathrm{H}$, pyridine- $\mathrm{H}), 8.09$ (d, $J=8.5 \mathrm{~Hz}, 1 \mathrm{H}, \mathrm{Ph}-\mathrm{H}), 7.62$ (d, $J=2.0 \mathrm{~Hz}, 1 \mathrm{H}, \mathrm{Ph}-\mathrm{H}$ ), 7.44 (dd, $J=8.5,2.0 \mathrm{~Hz}, 1 \mathrm{H}, \mathrm{Ph}-\mathrm{H}) ;{ }^{19} \mathrm{~F}$ NMR (471 MHz, chloroform-D) $\delta-62.46 ;{ }^{13} \mathrm{C}$ NMR (125 MHz, chloroform-D) $\delta$ 163.52, 161.66, 144.78, 143.86, 138.94, 136.78, 134.49, 132.41, 132.36, 131.46, $129.02(\mathrm{q}, J=34.0 \mathrm{~Hz}), 127.87,122.16$ (q, $J=273.5 \mathrm{~Hz}), 121.15$; HR-MS (ESI): calculated for $\mathrm{C}_{14} \mathrm{H}_{5} \mathrm{Cl}_{3} \mathrm{~F}_{3} \mathrm{~N}_{3} \mathrm{O}[\mathrm{M}+\mathrm{H}]^{+}$: 393.95231 , found: 393.95184 .

\section{Insecticidal activity}

The insecticidal activity was tested at $25 \pm 1{ }^{\circ} \mathrm{C}$ according to statistical requirements. Mortalities were calculated and based on a percentage scale using Abbott's formula. ${ }^{41}$

Insecticidal activity against oriental armyworm. The insecticidal activity against oriental armyworm was investigated using reported procedure. ${ }^{38}$ The corn leaves were put on the moistened filter paper in a Petri dishes (diameter $9 \mathrm{~cm}$ ) and sprayed with the solution containing the tested compounds. After drying, 10 fourth instar oriental armyworm larvae were then transferred to the dishes. Mortalities were calculated after treatment for 4 days. Commercial avermectin was also tested under the same conditions. Three replicates and at least five concentrations were performed for each experiment for $\mathrm{LC}_{50}$.

Insecticidal activity against $\boldsymbol{P}$. xylostella. The insecticidal activities against $P$. xylostella were evaluated using previously procedure. ${ }^{39}$ Fresh cabbage discs were dipped into the prepared solutions and placed in a Petri dish (diameter $9 \mathrm{~cm}$ ) lined with moistened filter paper. Fifteen larvae of second instar P. xylostella were carefully transferred to the Petri dish and cultivated for $72 \mathrm{~h}$. Commercial chlorpyrifos was tested under the same conditions as positive controls, four replicates were performed for each experiment at different concentration.

Insecticidal activity against Nilaparvata lugens and Aphis craccivora. Insecticidal activities against $N$. lugens and A. craccivora were tested according to our using the Potter spray method. ${ }^{41}$ Forty third-instar larvae of $N$. lugens (or A. craccivora) were treated at corresponding concentration at a pressure of $4.35 \mathrm{mg} \mathrm{cm}^{-2}$ under a Potter spray tower. Mortalities were then counted after rearing for 72-96 h. The treatments of imidacloprid and water were conducted as controls. Four replicates were measured for each treatment.

\section{D-QSAR study}

3D-QSAR study was performed according to our provirus work ${ }^{\mathbf{4 2}}$ using Sybyl 2.0X software (Tripos Inc., St Louis, MO). All the structures were drawn using the SKETCH option and minimized using the Gasteiger-Hückel charge, tripos force field and Powell conjugate gradient algorithm with a convergence criterion of $0.05 \mathrm{kcal} \mathrm{mol}^{-1}$. Then lowest energy conformation was determined using protocol of genetic algorithm (GA) conformational searches using the default setting in Sybyl. The structures were then aligned on a common substructure with compound E20 as the template molecule (Fig. S1 in ESI $\dagger$ ).
Compounds E7, E8, E9, E16, E22 and E26 were randomly chosen as a test set. The results of alignment are shown in Fig. S1, B (ESI $\dagger$ ). Then, CoMFA was performed using the partial least-squares (PLS) protocols to yield all the corresponding parameter (see Table S1 in ESI $\dagger$ ). CoMSIA analysis could be done in the similar PLS protocol as CoMFA.

\section{Conflicts of interest}

There are no conflicts to declare.

\section{Acknowledgements}

This work was supported by the National Natural Science Foundation of China (No. 21762012, 21562012), the Special Foundation of S\&T for Outstanding Young Talents in Guizhou (No. 2015-15\#) and the Innovative Foundation for Graduated Student of Guizhou University (No. 2017058).

\section{Notes and references}

1 E. C. Oerke, J. Agric. Sci., 2005, 144, 31-43.

2 H. X. Bian, H. F. Ma, X. X. Zheng, M. H. Peng, Y. P. Li, J. F. Su, H. Wang, Q. Li, R. X. Xia, Y. Q. Liu and X. F. Jiang, Sci. Rep., 2017, 7, 2324.

3 Z. Y. Li, X. Feng, S. S. Liu, M. S. You and M. J. Furlong, Annu. Rev. Entomol., 2016, 61, 277-296.

4 W. J. Kang, H. N. Koo, D. H. Jeong, H. K. Kim, J. Kim and G. H. Kim, Entomol. Res., 2017, 47, 394-403.

5 Y. Zuo, H. Wang, Y. Xu, J. Huang, S. Wu, Y. Wu and Y. Yang, Insect Biochem. Mol. Biol., 2017, 89, 79-85.

6 Y. Zhang, B. Yang, J. Li, M. Liu and Z. Liu, Insect Mol. Biol., 2017, 26, 453-460.

7 S. Zhang, X. Zhang, J. Shen, D. Li, H. Wan, H. You and J. Li, Pestic. Biochem. Physiol., 2017, 140, 85-89.

8 P. A. Umina, A. Lord, S. Micic and O. Edwards, Pest Manage. Sci., 2017, 73, 1719-1724.

9 H. X. Deng, Zhejiang Chem. Ind., 2011, 42, 1-3.

10 P. J. M. Jung, A. Edmunds, A. Jeanguenat and M. Muehlebach, Chem. Abstr., 2016, 164, 507520.

11 P. J. M. Jung, A. Edmunds, M. Muehlebach and R. G. Hall, Chem. Abstr., 2017, 167, 44589.

12 I. Yonemura, A. Suwa and S. Matsuo, Chem. Abstr., 2016, 164, 394066.

13 A. Edmunds, M. Muehlebach, P. J. M. Jung and A. Jeanguenat, Chem. Abstr., 2016, 165, 212702.

14 I. Yonemura, A. Suwa, S. Matsuo and T. Aoki, Chem. Abstr., 2016, 165, 247300.

15 H. Hiroki, Y. Kobayashi, J. Suzuki, S. Onoue, K. Iwasaki, A. Ootaka, H. Doi, E. Matsuo, M. Onoue and A. Okimoto, Chem. Abstr., 2017, 167, 68507.

16 J. M. Babcock, C. B. Gerwick, J. X. Huang, M. R. Loso, G. Nakamura, S. P. Nolting, R. B. Rogers, T. C. Sparks, J. Thomas, G. B. Watson and Y. Zhu, Pest Manage. Sci., 2011, 67, 328-334.

17 M. Bachwani, V. Sharma and R. Kumar, Int. Res. J. Pharm., 2011, 2, 84-89. 
18 Y. Zhou, B. Wang, F. Di, L. Xiong, N. Yang, Y. Li, Y. Li and Z. Li, Bioorg. Med. Chem. Lett., 2014, 24, 2295-2299.

19 T. P. Mohan, B. Vishalakshi, K. S. Bhat, K. S. Rao and G. N. Kendappa, Indian J. Chem., Sect. B: Org. Chem. Incl. Med. Chem., 2004, 43, 1798-1801.

20 Q. Liu, K. Chen, Q. Wang, J. Ni, Y. Li, H. Zhu and Y. Ding, RSC Adv., 2014, 4, 55445-55451.

21 D. He and H. Lu, Chem. Abstr., 2011, 160, 117899.

22 D. He and X. Li, Chem. Abstr., 2011, 155, 352554.

23 Y. Guo, L. Qu, X. Wang, M. Huang, L. Jia and Y. Zhang, RSC Adv., 2016, 6, 93505-93510.

24 H. Dai, Y. Shi, H. He, Y. Li, Z. Jin, Y. Xiao, Y. Yuan and Y. Fang, Chem. Abstr., 2016, 165, 223979.

25 W. Xu, J. He, M. He, F. Han, X. Chen, Z. Pan, J. Wang and M. Tong, Molecules, 2011, 16, 9129-9141.

26 S. Shen, X. Sun, Y. Liu, B. Chen, R. Jin and H. Ma, J. Heterocycl. Chem., 2015, 1296-1301.

27 S. Shen, X. Sun, Y. Liu, B. Chen, R. Jin and H. Ma, Chem. J. Chin. Univ., 2014, 35, 1427-1432.

28 A. K. Mittal, D. V. Singh and S. Tripathi, J. Chem., Biol. Phys. Sci., 2012, 2, 699-707.

29 J. Liu, W. Wang and H. He, Chin. J. Org. Chem., 2014, 34, 1447-1451.

30 Z. N. Cui, Y. X. Shi, L. Zhang, Y. Ling, B. J. Li, Y. Nishida and X. L. Yang, J. Agric. Food Chem., 2012, 60, 11649-11656.
31 J. Wu, S. Kang, L. Luo, Q. Shi, J. Ma, J. Yin, B. Song, D. Hu and S. Yang, Chem. Cent. J., 2013, 7, 64.

32 Q. Mo, W. Duan, X. Li, D. Huang and Z. Luo, Chin. J. Org. Chem., 2011, 31, 1114-1121.

33 M. Kalhor and A. Dadras, J. Heterocycl. Chem., 2013, 50, 220224.

34 W. G. Duan, X. R. Li, Q. J. Mo, J. X. Huang, B. Cen, X. T. Xu and F. H. Lei, Holzforschung, 2011, 65, 191-197.

35 Y. Guo, L. Qu, X. Wang and M. Huang, Chem. Abstr., 2016, 166, 283974.

36 Y. Y. Wang, X. M. Lu, J. Shi, J. H. Xu, F. H. Wang, X. Yang, G. Yu, Z. Q. Liu, C. H. Li, A. L. Dai, Y. H. Zhao and J. Wu, Monatsh. Chem., 2018, DOI: 10.1007/s00706-017-2060-3.

37 P. Li, L. Shi, X. Yang, L. Yang, X. W. Chen, F. Wu, Q. C. Shi, W. M. Xu, M. He, D. Y. Hu and B. A. Song, Bioorg. Med. Chem. Lett., 2014, 24, 1677-1680.

38 Q. Q. Zhao, J. Shang, Y. X. Liu, K. Wang, F. C. Bi, R. Huang and Q. M. Wang, J. Agric. Food Chem., 2007, 55, 559614559619.

39 J. H. Xing, W. G. Cai, S. R Wang, J. Chen, S. T. Zhang, R. X. Fu and Q. M. Fu, Plant Prot. Sci., 2007, 33, 116-118.

40 C. Potter, Ann. Appl. Biol., 1952, 39, 1-28.

41 W. S. Abbott, J. Am. Mosq. Control Assoc., 1987, 3, 302-303.

42 J. Wu, B. A. Song, D. Y. Hu, M. Yue and S. Yang, Pest Manage. Sci., 2012, 55, 801-810. 